\title{
¿Cuáles son los Rasgos que Han de Caracterizar el Perfil de los Egresados de las Escuelas de Negocios Jesuitas Según sus Directivos?
}

\author{
Enrique López Viguria ${ }^{1 *}$, Ricard Santomà ${ }^{2}$
}

\begin{abstract}
This article aims to detect a central element in the mission of Spain Jesuit Universities Business Schools (UNIJES). In particular, it focuses on the analysis of the features that have to characterize the profile of alumni from these schools.
\end{abstract}

To achieve this goal, we use the Concept Mapping methodology to identify ideas and opinions from a group of experts (raw data from focus group) and to transform them into classified data displayed in conceptual maps. The results show how the universities governments value the traits in the alumni. The four most important detected aspects are (i) professional skills, (ii) intrapersonal skills, (iii) interpersonal skills and (iv) social responsibility. These aspects are analyzed from the perspective of the education paradigm within Jesuit Universities.

Keywords: Mission; bussiness school; alumni trait; conceptual map

Resumen: El objetivo de este artículo es estudiar un elemento central de la misión de las escuelas de negocios de UNIJES (Universidades Jesuitas en España), concretamente: cuáles son los rasgos que han de caracterizar el perfil de los egresados de dichas escuelas a juicio de las personas con responsabilidades de gobierno (rectores y directores).

Para ello, se utilizará la metodología del Concept Mapping, técnica que permite transformar las ideas/opiniones de un grupo de expertos (datos primarios de un focus group) en datos que pueden ser ponderados, agrupados y representados mediante mapas conceptuales. Los resultados revelan cómo se valoran y ponderan los rasgos del perfil de los egresados por parte de los citados directivos, y cómo se agrupan en cuatro clústeres: competencias profesionales (1), competencias intrapersonales (2), competencias interpersonales (3) y responsabilidad social /sentido de trascendencia (4), que son analizados e interpretados y que, además, permiten una visión comparativa con el paradigma educativo de las universidades jesuitas (Ledesma-Kolvenbach).

Los resultados de esta investigación pueden considerarse como una aportación original y novedosa acerca del pensamiento y valoración de autoridades académicas de este nivel sobre un aspecto esencial para las escuelas de negocios jesuitas, dado su carácter de instituciones mission-driven. La metodología utilizada también es una novedad en el sector de la educación universitaria y de las escuelas de negocios.

Palabras clave: misión; escuelas de negocios; rasgos del egresado; mapa conceptual

$21^{\text {st }}$ International Association of Jesuit Business Schools (IAJBS)

$18^{\text {th }}$ Colleagues in Jesuit Business Education (CJBE)

\section{Introducción}

En el contexto de un mundo globalizado que ha experimentado en estos últimos años una importante crisis financiera y económica, especialmente en Occidente, se ha puesto en cuestión el papel y el liderazgo, los valores y la responsabilidad, de los directivos de muchas entidades financieras, instituciones públicas y grupos multinacionales.

Este cuestionamiento ha propiciado una revisión sobre la misión y el propósito de la educación superior y de las universidades. Este debate se ha extendido, por razones obvias, al ámbito de las escuelas de negocios (en adelante BS, por sus siglas en inglés, Business Schools) acerca de su papel y su contribución real en la formación de líderes y directivos, de sus modelos pedagógicos y sus presupuestos axiológicos. $\mathrm{Y}$ este debate invita a las escuelas han de realizar una profunda reflexión con el propósito de darle un nuevo sentido "a su identidad y crear un claro sentido de propósito" (Starkey y Tempest, 2008) y porque, como apunta Khurana (2007), la business education puede y debe ser una experiencia transformadora.

Algunos autores e instituciones muy vinculadas a las BS han subrayado que "no se trasmiten valores ni se educa a través de lo que se dice, sino a través de lo que se hace y se es" (Losada, Martell y Lozano, 2011). De ahí, la importancia de desarrollar la misión e identidad de las BS en claves de ética y responsabilidad social (PRME, 2008; GRLI, 2010; AACSB International, 2015).

En este contexto, es pertinente dirigir la mirada hacia una de las instituciones mundiales con mayor protagonismo en este ámbito formativo, la Compañía de Jesús (en adelante, SJ), con una de las mayores

(1) ESADE Business School, Universidad Ramon Llull.

(2) Facultad de Turismo y Dirección Hotelera Sant Ignasi, Universidad Ramon Llull

${ }^{*}$ Corresponding autor: enrique.lopez@esade.edu 
redes de universidades del mundo, así como de escuelas de negocios, la International Association of Jesuit Business Schools (en adelante IAJBS).

Del debate suscitado se hacía eco el Superior General de la SJ cuando invitaba a "los colegas de las aproximadamente 200 instituciones de educación superior que funcionan bajo la bandera de la Compañía de Jesús", a "reflexionar juntos sobre cómo este nuevo contexto nos plantea el desafío de reorientar, en cierto sentido, la misión de la educación jesuita” (Nicolás, 2010).

En el ámbito universitario, la misión intelectual de la SJ se ha ido estableciendo a través de lo dispuesto en sus Congregaciones Generales y de lo expresado en los principales discursos de sus Superiores Generales. Ambas fuentes han inspirado en su misión y razón de ser a sus universidades, que han realizado la adaptación consiguiente según sus circunstancias particulares y, en modo similar, las escuelas de negocio de la SJ (en adelante JBS, por sus siglas en inglés, Jesuit Business Schools), vinculadas habitualmente al ámbito universitario.

Por todo ello, se importante conocer y analizar la misión de la SJ y de sus instituciones universitarias y JBS, y, teniendo en cuenta que una de las dimensiones clave de la misma es la formación de personas, establecer en este estudio cuáles son los rasgos que han de caracterizar el perfil del egresado de sus escuelas de negocios, a juicio de sus máximos responsables en el ámbito español de UNIJES (Universidades Jesuitas, España).

Para ello, se usará la herramienta del concept mapping (Trochim, 1989) cuya principal ventaja radica en que permite ordenar de manera sistemática los resultados obtenidos de un focus group de expertos, mediante el brainstorming y la combinación de técnicas estadísticas como son el escalamiento multidimensional y el análisis clúster (Bigné, Aldás-Manzano, Küster y Vila, 2002).

Para alcanzar los objetivos del estudio, previamente se lleva a cabo una revisión sintética de la literatura sobre la misión en el ámbito general de las empresas y organizaciones, para particularizar después la atención con mayor detalle sobre la misión de la SJ, sus universidades y escuelas de negocios.

\section{La misión en el ámbito de las empresas, organizaciones y la educación superior. La misión para la compañiade jesús, sus universidades y escuelas de negocios.}

La misión expresa a qué se dedica una organización, cuál es su propósito, qué es lo que persigue. La misión enmarca la finalidad de la organización y, de alguna forma, declara el sentido de su propia existencia, su razón de ser (David, 1989), articulando la filosofía de la empresa (propósito y valores) con la visión de futuro de la misma (Collins y Porras, 1996).

En la literatura revisada es muy frecuente citar a Peter F. Drucker como el autor que introduce, en la década de "los setenta", el tema de la misión en la agenda del análisis y de la estrategia de las empresas y organizaciones. Una clara definición de la misma, de su propósito, hará posible la fijación de objetivos claros y realistas (Drucker, 1973). Diversos autores de manuales de referencia en este campo han subrayado la importancia de la misión como pieza fundamental a la hora de analizar, planificar e implementar la estrategia empresarial (e.g., Johnson y Scholes, 1996; Guerras y Navas, 2007).

Según sea el enfoque del papel que juega la misión en las organizaciones, se pueden distinguir dos líneas de pensamiento. La primera, concibe la misión en términos de estrategia de negocio y, en este sentido, es una pieza clave (strategic tool) en la planificación estratégica Pearce, 1982). La segunda línea de pensamiento, que puede ser complementaria a la anterior, concibe la misión en términos de filosofía y ética, como un elemento clave para dotar de propósito, valores y cultura (cultural glue) a las organizaciones (Campbell y Yeung, 1991).

La concepción de la misión en la educación superior es deudora de la literatura generada sobre este tema en el ámbito del management, difiriendo poco el concepto de misión del utilizado en el campo empresarial (Edem, Spencer y Fyfield, 2003). Como en el mundo de las empresas, también en el ámbito de la educación superior resultaba clave su uso para los procesos de planificación estratégica (Davies y Glaister, 1997).

En los últimos años, su importancia ha ido en aumento y un aspecto que ha favorecido su impulso han sido los procesos de acreditación y aseguramiento de la calidad. En este sentido, hay que reseñar la importancia concedida a la misión en el ámbito de las BS por parte de organismos de acreditación como la Association to Advance Collegiate Schools of Business (AACSB) y The European Quality Improvement System (EQUIS) Concretamente, como señalan Palmer y Short (2008) refiriéndose a la AACSB, "la gestión del proceso estratégico tiene su corazón en la misión” de las BS. (2008, p. 456).

Para la AACSB hay tres criterios relacionados con la misión de las BS que tienen una importancia crítica, y que refuerzan su orientación misional (mission driven): primero, la misión debe ser adecuada, descriptiva y transparente para los stakeholders de la escuela; segundo, la misión debe proveer a la escuela de una dirección clara para la toma de decisiones; y en tercer lugar, las estrategias de la escuela y los resultados esperados deben estar alineados con la misión (AACSB International, 2015, p. 13).

\section{La Compañía de Jesús y su Misión.}

La palabra "misión" tiene connotaciones distintas cuando se utiliza en el contexto del management (especialmente referido a las empresas en el mercado) y en el contexto de la SJ (referido a una visión religiosa del mundo). Ciertamente, hay relación entre ambos contextos, y se puede analizar la SJ como una organización fuertemente orientada a la misión (mission-driven), pero requiere algunas precisiones conceptuales. Para la SJ, la concepción de la misión se aproxima a la misión de las organizaciones en el sentido de identidad, razón de ser y valores, y, claramente en menor medida, como pieza estratégica. Dicho de otro modo, analizada la misión de la SJ desde la perspectiva del 
management, se consideraría como axiológicamente "sobrecargada". Por eso, la misión de la SJ no se pude analizar solamente en clave profesional, de gestión o de estrategia organizativa, porque se mueve en una perspectiva teológica, y también en una perspectiva existencial.

La sobrecarga axiológica citada y la idea del compromiso existencial también alcanza a la razón de ser y a la misión de las instituciones universitarias y las JBS, que han de buscar la manera concreta en su contexto de llevar a cabo del mejor modo posible su propia misión, su razón de ser, en consonancia con la misión de la SJ. Por tanto, es necesaria una aproximación "cultural" más que estratégica al estudiar una institución como la SJ, fuertemente orientada por su misión, que ya en los documentos de la época fundacional (1540) reflejaba el propósito y la vocación de los fundadores, Ignacio de Loyola y su grupo de compañeros, de constituirse como un "cuerpo", cuya unidad sólo tiene sentido por su carácter misionero (Salvat, 2001, p. 175).

La misión o el fin de la Compañía de Jesús se ha expresado de diversas formas a lo largo de la historia. Numerosos autores han estudiado la vida del fundador, Ignacio de Loyola, así como la historia de la SJ y de sus protagonistas, su misión, espiritualidad y desarrollo en el marco de la Iglesia católica, así como su extraordinaria influencia (Guichard, 1974; Woodrow, 1984; Lacouture, 1993; O’Malley, 1993; Salvat, 2001; Lowney, 2004; Martin, 2011).

Desde la aprobación de la SJ, con la Bula de Paulo III "Regimini militantis Ecclesiae", que establece la primera fórmula del nuevo Instituto, y posteriormente en Las Constituciones y en las sucesivas Congregaciones Generales (en adelante CG), se ha ido concretando la misión de la SJ cuyo objetivo central y fundamental es el servicio de la fe para ayudar a las ánimas (a las personas).

Cabe destacar que en la CG 32 (1975, p.74), en su decreto IV, se definía la misión de la Compañía como "el servicio de la fe, del cual la promoción de la justicia es una exigencia absoluta”, una declaración decisiva en las formulaciones y en la práctica posterior. Más adelante, en la CG 34 (1995, p. 83), se amplió esta dimensión afirmando, "nuestra misión de servicio de la fe y promoción de la justicia (principio integrador) debe ensancharse para incluir como dimensiones esenciales la proclamación del Evangelio, el diálogo, y la evangelización de la cultura".

El documento pedagógico más relevante históricamente de la SJ, es conocido por su título abreviado de Ratio Studiorum, y fue "el reglamento de estudios obligatorio, estable y definitivo" hasta finales del siglo XVIII (Gil Coria, 1999), así como un currículo básico y modo de proceder para los colegios y universidades jesuitas hasta después de la Segunda Guerra Mundial. (Margenat, 2010). Posteriormente, al acercarse al cuarto centenario de la Ratio Studiorum, la SJ producirá dos documentos que actualizan la herencia ignaciana con una propuesta educativa para los nuevos tiempos: Características de la Educación de la Compañía de Jesús (1986) y Pedagogía Ignaciana, un planteamiento práctico (1993), citados por Gil Coria, E. (1999).
La misión de las universidades jesuitas y de sus escuelas de negocios.

Acerca de la misión intelectual en la universidad, la CG 34 (1995) subraya su importancia y la necesidad de "clarificar su núcleo esencial, dada la diversidad de sus formas en el ancho mundo". Concretamente, la SJ apunta las siguientes prioridades de este núcleo esencial:

- Las instituciones han de mantener y fortalecer su carácter específico en cuanto universidades (sustantivo) y en cuanto jesuíticas (adjetivo). El sustantivo garantiza el compromiso con la autonomía fundamental, la integridad y la sinceridad de una universidad, en cuanto tal. En cuanto jesuíticas, se ha de interrogar acerca del "para qué del conocimiento" (CG 34, 1995, p.346).

- La universidad jesuítica ha de promover en sus objetivos y dinámica institucional un marco adecuado para encarnar el "servicio de la fe que obra la justicia, en línea con la misión de la Compañía, descubriendo nuevos campos y horizontes de investigación, enseñanza y extensión universitaria (CG 34, 1995, p. 348-9).

- Las universidades de la SJ han de distinguirse por orientar su quehacer docente e investigador desde una misión e identidad integrada por cuatro dimensiones fundamentales, en lo que se ha dado en llamar el paradigma o modelo Ledesma-Kolvenbach, utilizando las expresiones latinas: Utilitas, Humanitas, Iusticia y Fides. Dicho en otros términos, con una visión integral y holística del "para qué" universitario, ofreciendo una formación práctica / profesional, humana, social, moral y religiosa (Agúndez, 2008a).

En los principales discursos y conferencias de los Superiores Generales (Arrupe, 1981 y 1983; la recopilación de los discursos universitarios de Kolvenbach, en Agúndez, 2008; Nicolás, 2008 y 2010), acerca del rol y la de la misión de los centros superiores y de las universidades, se evidencia la importancia que la SJ ha concedido a este tipo de instituciones desde sus orígenes, y su relevancia cultural, social y apostólica en el presente. En este sentido, P. H. Kolvenbach señalaba en la presentación de su recopilación de discursos universitarios, "me atrevería a afirmar que la universidad jesuítica ha nacido "ex corde Societatis”--del corazón de la Compañía-- (Agúndez 2008a). Estos discursos y conferencias se pronuncian ante diversos auditorios y con motivo de circunstancias particulares, con diversas formulaciones e implicaciones de la misión intelectual y universitaria de la SJ. Sus contenidos, de alguna manera, son una interpretación y actualización "autorizadas" de la misión de la SJ en este ámbito, al provenir de su Superior General (en adelante, SG).

Cabe citar también el último Encuentro Mundial de Rectores de Universidades Jesuitas, (México, 2010), en el que el SG, A. Nicolás, se refirió a los desafíos de la educación superior jesuita hoy, invitando a reflexionar sobre el nuevo contexto de la globalización, que "plantea el desafío de que la educación superior jesuita redefina o, por lo menos, reoriente su misión" (2010, p. 72). 
En el ámbito de la misión y los desafíos de las universidades y de las JBS, se pueden destacar algunos documentos y estudios de referencia, tales como: Jesuit Business Schools: four reflections (Delbeq et al, 1983); un estudio pionero de Berleur, Harvanek y Corliss (1997), sobre la MS en las instituciones de educación superior y universidades jesuitas; y un estudio posterior (Mussi, 2008) que exploró, en las universidades jesuitas, la relación existente entre la misión y el trabajo que realiza el staff. También se vienen presentando estos desafíos misionales en las conferencias anuales de IAJBS, red de JBS ya cita$\mathrm{da}$, en las reuniones anuales de CJBE (Colleagues of Jesuit Business Education), que publica el Journal of Jesuit Business Education desde del verano de 2010, y en los encuentros y publicaciones de UNIJES, de AUSJAL (red universitaria de la SJ en América Latina) y en otras redes universitarias de la SJ. Existen múltiples documentos y artículos referidos a la misión universitaria y en las JBS, presentados en jornadas interuniversitarias y conferencias anuales de las redes mencionadas más arriba, publicados en revistas y journals, cuya relación excede el alcance de este artículo.

\section{El modelo universitario Ledesma-Kolvenbach.}

El ya citado modelo Ledesma-Kolvenbach es una referencia obligada al tratar acerca de la misión de las universidades y de las JBS. Es una expresión acuñada por el jesuita Melecio Agúndez, cuando afirma: "hemos decidido designarle con este nombre compuesto porque, si bien la estructura cuatridimensional es original de Ledesma (s.XVI), la traducción a lenguaje moderno y la terminología latina acuñada para Georgetown-Gregoriana (utilitas, iustitia, humanitas, fides) es producto made in Kolvenbach" (Agúndez, 2008a, p. 24).

Diego de Ledesma fue un teólogo y pedagogo jesuita que, en el siglo XVI, tuvo una gran influencia en la creación de la Ratio Studiorum, y fue quien respondió por vez primera" a la pregunta de por qué la Compañía de Jesús debería mantener instituciones educativas y lo hizo con una fórmula "cuatridimensional" con estas palabras (Agúndez, 2008a, p. 258 y 259):

"Lo primero, porque proveen a la gente con muchas ventajas para la vida práctica; en segundo lugar, porque contribuyen al correcto gobierno de asuntos públicos y a la apropiada formulación de leyes; en tercer lugar, porque dan decoro, esplendor y perfección a nuestra naturaleza racional; y en cuarto lugar, que es de suma importancia, porque son la defensa de la religión y nos guían con gran seguridad y facilidad en la consecución de nuestro fin último”.

P. H. Kolvenbach (SG de la SJ, 1983-2008) utiliza la referencia a Ledesma y la "traduce" al mundo universitario de la SJ principalmente en su discurso dirigido al Consejo Directivo de la Universidad de Georgetown, en una reunión celebrada en Roma en 2007. A continuación, parafraseando a Kolvenbach sobre éste y otros discursos en que alude al modelo citado, se destacan algunos aspectos principales de las cuatro dimensiones:
Utilitas: la finalidad práctica de la universidad, con la mira puesta en asegurar los conocimientos y competencias con los que sobresalir en el campo de especialización elegido.

Iustitia: educar mujeres y hombres de modo que puedan abrazar y promover todo lo que debe hacerse para construir unas justas estructuras sociales, económicas y políticas que defiendan nuestra humanidad común y una paciente promoción de la justicia.

Humanitas: la formación de personas más plenamente humanas desde el credo y tradición humanísticos de la educación jesuita, la libertad ilustrada de la conciencia, la libertad responsable de la palabra, el diálogo respetuoso y una solidaridad bien informada.

Fides: la dimensión religiosa; en su más profundo sentido, la entrega a la búsqueda de la plenitud de la verdad y, en este sentido, la universidad jesuita debe proponer la fe cristiana y ayudar a todo ser humano a encontrar al Señor.

Para Kolvenbach, lo importante es responder a una pregunta que él formula con cierta frecuencia: ¿qué tipo de hombres y mujeres necesitamos formar para que sean los líderes del tercer milenio? Por ello, el modelo Ledesma-Kolvenbach plantea el "para qué" de la educación universitaria jesuita con la pretensión de formar a la "persona completa", señalando cuatro dimensiones fundamentales e interdependientes, "como cuatro aspas de un hélice" (Agúndez, 2008b), que resumen el conjunto de rasgos que han de perfilar a quienes pasen por esta formación. Y esta pretensión, esta visión holística e integral, solamente puede fructificar en cómo sean y qué hagan los egresados. Por ello, para Kolvenbach "el auténtico criterio para evaluar las universidades de la Compañía no es lo que nuestros estudiantes hagan, sino lo que acaben siendo y la responsabilidad cristiana adulta con la cual trabajen en el futuro a favor de sus prójimos y de su mundo" (Kolvenbach, 2008, p. 183).

El modelo Ledesma-Kolvenbach ha sido mencionado y reinterpretado por A. Nicolás, actual SG de la SJ, en varios de sus discursos, haciéndose eco también de otra forma de aproximación a este contenido, más concreta desde el punto de vista de las características o rasgos de los egresados, cuando dice: "en estos últimos tiempos, los jesuitas y los laicos implicados en la educación universitaria hablan de cuatro características de la persona humana íntegra e integral, a partir de cuatro cualidades que empiezan por la letras "C". En efecto, el espíritu humanista genera personas conscientes, competentes, compasivas y comprometidas". (Nicolás, 2008).

\section{Enfoque del estudio y metodología utilizada}

La revisión de la literatura presentada en el apartado anterior nos ha permitido conocer y analizar el concepto de misión en general y, más particularmente, en el ámbito de la SJ y de sus universidades y JBS. 
Teniendo en cuenta este análisis, y cómo se concreta en el ya citado paradigma Ledesma-Kolvenbach, se constata que una dimensión clave de la misión de la SJ es la formación de personas. Por ello, el objeto de este estudio se enfoca a conocer cuáles son los rasgos que han de caracterizar el perfil del egresado de las JBS, según el criterio de sus máximos responsables.-

Este estudio utilizará la herramienta metodológica del concept mapping (Trochim, 1989) cuya principal ventaja radica en que permite ordenar de manera objetiva los resultados obtenidos de un focus group de expertos y con el uso combinado de técnicas estadísticas como son el escalamiento multidimensional y el análisis clúster (Bigné et al, 2002).

El concept mapping (en adelante, CM) es una metodología de investigación poco utilizada hasta la fecha en este ámbito, pero que ha sido validada mediante su aplicación en otras áreas, como son la educación, la investigación social y el management, así como en diversos estudios y artículos de revistas académicas (Santomà, 2008).

En el momento de planificar o evaluar un proyecto, el paso más difícil es el de la conceptualización. Trochim (1989), creador del CM, la define como la representación objetiva de pensamientos, ideas, intuiciones y afirma que en muchas ocasiones es necesario conceptualizar medidas, resultados que se consideran importantes, ideas, muestras, etc. Según el autor, el CM puede dar respuesta a las necesidades de conceptualización y ofrecer al tiempo una metodología para validar el constructo de la investigación. Bigné et al. (2002) afirman que la técnica del CM es capaz de estructurar un proceso de conceptualización, objetivando los resultados de un focus group, representando las ideas expresadas en un mapa conceptual y, opcionalmente, ayudando a destacar las más importantes, relevantes o apropiadas. Para otros autores (Bigné et al., 2002; Rosas y Camphausen, 2007; Nabitz, Severens, Brink y Jansen, 2007), el CM es un proceso útil para elaborar el mapa conceptual de las ideas expresadas por un grupo de expertos.

El desarrollo de la técnica del CM obliga a cubrir seis etapas (Trochim, 1989):

\begin{tabular}{|c|}
\hline Figura 1: Proceso del Concept mapping \\
\hline Fase 1: Preparación o planificación \\
\hline$\nabla$ \\
\hline Fase 2: Generación de ideas \\
\hline$\downarrow$ \\
\hline Fase 3: Estructuración de las ideas \\
\hline $\boldsymbol{\gamma}$ \\
\hline Fase 4: Representación de las ideas \\
\hline $\boldsymbol{\nabla}$ \\
\hline Fase 5: Interpretación de los mapas \\
\hline$\nabla$ \\
\hline Fase 6: Utilización de los mapas \\
\hline Fuente: Trochim, (1989) \\
\hline
\end{tabular}

Los resultados del CM permitirán un análisis e interpretación de los mapas conceptuales y los clústeres de rasgos obtenidos, así como su comparación con el modelo Ledesma-Kolvenbach antes referido.

\section{Aplicación metodológica y resultados obtenidos}

Durante el año 2012 se aplicó la metodología del concept mapping mediante diferentes entrevistas y dos sesiones del mismo focus groups (mayo y noviembre). Las fases que se siguieron, indicadas por Trochim (1989) fueron:

\section{Fase 1: Preparación o planificación}

Tal como indica Tochim (1989), en esta fase del proceso deben desarrollarse dos aspectos; por un lado, debe clarificarse el constructo que se quiere investigar y, por otro, han de escogerse las personas que participarán en el proceso. Es recomendable que el grupo de participantes sea heterogéneo a fin de poder contar con diferentes puntos de vista.

Como trabajo preliminar, se realizaron cuatro entrevistas en profundidad no estructuradas con jesuitas con experiencia de gobierno a distintos niveles en la SJ y expertos en la materia del estudio, que tuvieron por objeto "el aprendizaje sobre lo que es importante en la mente de los informantes: sus significados, perspectivas y definiciones; el modo en que ellos ven, clasifican y experimentan el mundo." (Taylor y Bodgan, 1987, p.100), de cara a concretar cuál habría de ser la pregunta al focus group en el CM, aquélla que mejor sintetizara el propósito misional de las JBS.

Teniendo en cuenta la revisión de la literatura y las entrevistas realizadas, se planteó que para formular la pregunta sobre la misión de las JBS había que escoger una dimensión clave. Al tratarse de instituciones académicas, fundamentalmente con actividad formativa, aunque también con importante actividad en investigación y debate/ proyección social, se consideró que era la formativa la dimensión más relevante sobre la que preguntar y, concretamente, sobre las características o rasgos que deberían caracterizar a los egresados de este tipo de instituciones, pues las respuestas concretarían el sentido y propósito fundamental de las mismas.

A raíz de ello, se formuló así la pregunta de investigación:

Como responsables del gobierno de las instituciones universitarias y escuelas de negocios vinculadas a la Compañía de Jesús en España, en su opinión: ¿Qué rasgos han de caracterizar el perfil de los egresados de sus escuelas de negocios?

Los criterios para la selección de los expertos del focus group --entre ocho y doce, según recomienda Trochim (1989)-- fueron los siguientes: personas con experiencia en la dirección en este tipo de instituciones y/o en el gobierno de la Compañía de Jesús, o en su sector universitario. Por ello, se escogió un grupo que reúne estos criterios: la Junta de UNIJES, órganos directivo en el que se integran los once rectores y directores de los centros de esta red de instituciones universitarias vinculadas a la Compañía de Jesús en España, y entre las cuales existen cinco escuelas de negocios: Deusto Business School (Universidad de Deusto); ICADE - ICADE Business School (Universidad Pontifcia Comillas); IQS School of Management (Instituto Químico de Sarriá - URL); ESADE Business School (ESADE - URL); Loyola Leadership School (Universidad 
Loyola Andalucía). El apoyo del director de UNIJES fue clave para facilitar las dos sesiones de focus group, programando un tiempo adicional en dos reuniones del órgano citado.

\section{Fase 2: Generación de ideas}

Una vez superado el primer paso, se invitó a los participantes a generar ideas relacionadas con el tema central. La técnica que se utilizó fue la del brainstorming, siguiendo las normas de funcionamiento de Osborn (1948) y Dunn (1982), y contando con la colaboración de un experto para realizar las dinámicas de grupo con el fin de obtener unos mejores resultados, como propone Bigné et al. (2002).

La sesión se desarrolló coincidiendo con una reunión de la Junta de UNIJES (Mayo de 2012). El brainstorming con los 11 expertos se centró en responder a la pregunta ya citada, generándose una lista de 103 ítems o rasgos sobre el perfil de los egresados, lista que se aceptó como definitiva después de ser revisada.

\section{Fase 3: Estructuración de las ideas}

El propósito de esta fase consiste en determinar cómo están relacionadas entre sí las diferentes ideas surgidas en la fase anterior (Trochim 1989). Para ello, se solicitó a los participantes la realización de dos actividades: la primera, que valorasen la importancia de cada una de los rasgos fruto del brainstorming, puntuándolas de 1 a 5, en base a una escala de Likert (Bigné et al, 2002); y la segunda, la agrupación de los rasgos de la lista, según su propio criterio de similitud o relación (Trochim, 1989), asignando una etiqueta descriptiva con un nombre a cada uno de los grupos resultantes.

Una vez los participantes hicieron las valoraciones y agrupaciones se construyó una matriz con tantas filas y columnas como rasgos surgieron. En cada intersección se colocó un "1" si una persona del grupo había puesto los dos ítems en el mismo grupo o un "0" en caso contrario. A continuación las matrices individuales se agregaron para conseguir una matriz grupal (figura 2).

En la diagonal de la matriz de intersecciones figura el número total de participantes y el número de cada intersección muestra el número de personas que colocaron cada par de rasgos (R) en el mismo grupo independientemente del significado o criterio utilizado.

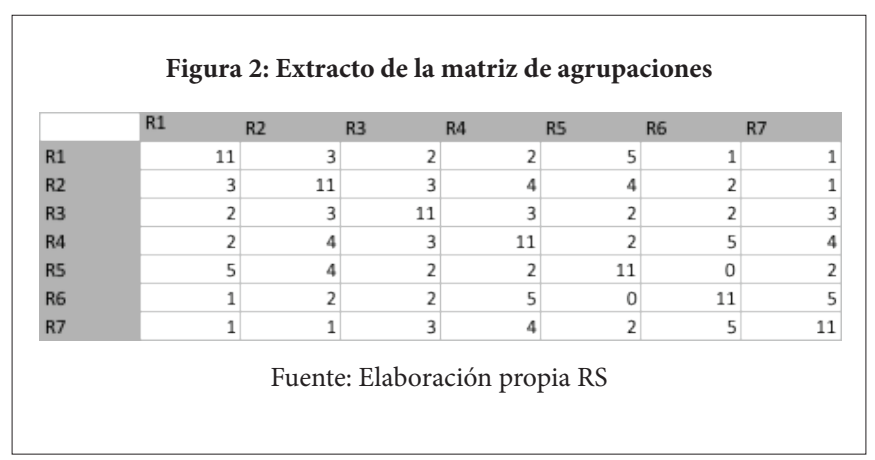

\section{Fase 4: Representación de las ideas}

Siguiendo las indicaciones de Trochim (1989), la conceptualización y representación gráfica de los resultados obtenidos se realizó aplicando técnicas estadísticas. En primer lugar la matriz de agrupaciones obtenida en la fase anterior se sometió a un escalado multidimensional (MDS), siguiendo el método Alscal, del que se obtuvo un mapa en el que cada punto representaba una idea. La mayor o menor cercanía entre los puntos debe entenderse como representativa del número de expertos que colocaron dichos rasgos en un mismo grupo.

Figura 3: Mapa de puntos resultante del Multidimensional Scaling

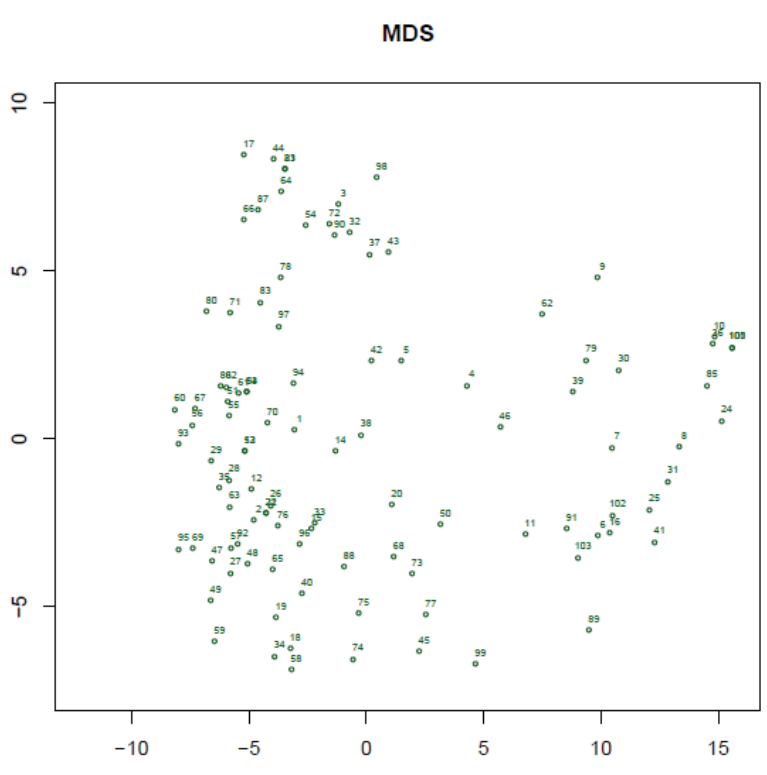

Fuente: Elaboración propia

Una vez construido el mapa representado en la figura 3, cuyos ejes de coordenadas reflejan escalas de distancias, se realizó un análisis clúster jerárquico (siguiendo el método Ward) utilizando las distancias entre los puntos a partir de sus coordenadas en el mapa resultante del MDS.

\section{Fase 5: Interpretación de los mapas}

En esta fase el objetivo es describir, analizar e interpretar los mapas obtenidos en la fase anterior (Trochim 1989). Obtenidos varios mapas con distinto número de clústeres, había que escoger cuál era el número óptimo de clústeres atendiendo a la mayor relación de significado entre los rasgos de cada clúster.

Para ello, se realizó una segunda sesión con el focus group (Junta de UNIJES, Noviembre de 2012), con el objeto de presentarles los resultados obtenidos aplicando el MDS, y que pudieran ver los mapas de rasgos obtenidos y las opciones que ofrecía el análisis clúster, para que pudieran determinar cuál era el número óptimo de clústeres según el sentido de sus agrupaciones de ideas y pudieran proponer sus denominaciones. La sesión de trabajo sirvió para validar el proceso seguido. En cuanto a la opción por un número de clústeres se dudó entre 4 ó 5 y en referencia a cómo denominarlos se hicieron varias propuestas. Por la premura de tiempo disponible, ambas cuestiones se dejaron en manos de los autores del estudio para que, por sí mismos y en consulta con expertos en la materia, pudiera determinar finalmente el número de clústeres y precisar la denominación de las etiquetas, sobre la base de su mayor coherencia de significado. 
$\mathrm{Al}$ no haber encontrado una sistemática que determinase un número de clústeres (Hair, F, Black, Babin, Anderson, \& Tatham,. 2006), se decidió establecer un número en el que las agrupaciones meramente estadísticas tuvieran también una significación conceptual coherente por las ideas contenidas en cada grupo, tal como han hecho otros autores (Nabitz et al., 2001; Rosas y Camphausen, 2007).

Por ello, teniendo en cuenta el análisis exploratorio realizado en el focus group y la recomendación de los expertos consultados (profesores universitarios), coincidentes con los autores del estudio, y siguiendo el criterio de mayor coherencia de significado de los grupos de rasgos, se determinó que el número óptimo era de 4 clústeres (figura 4).

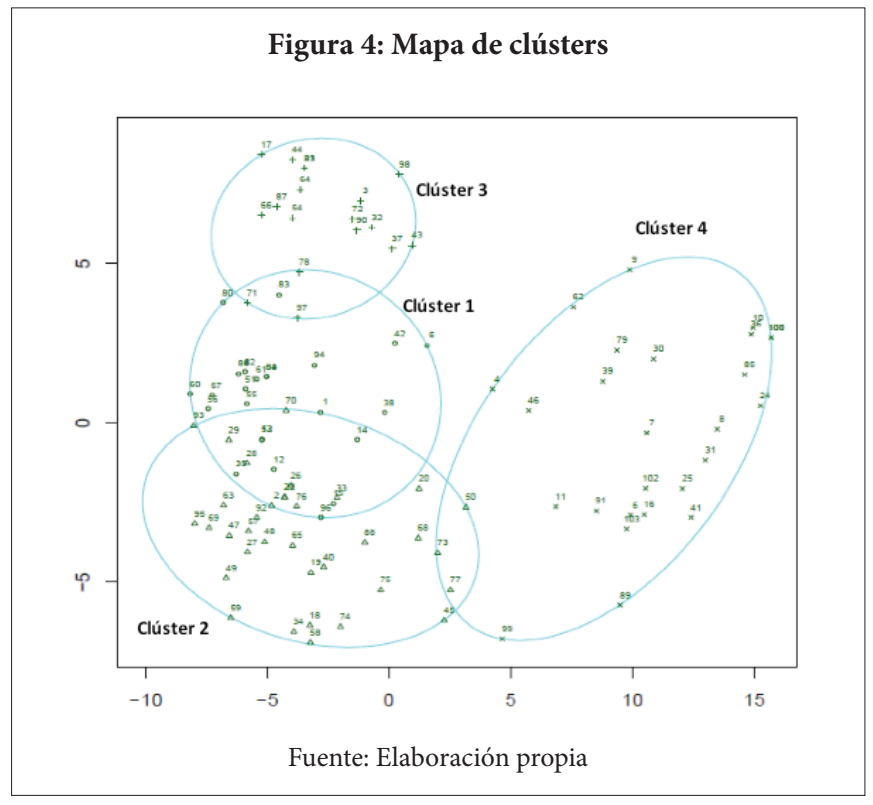

Así mismo, se decidió que no era necesario alterar la composición de los clústeres y que las etiquetas definitivas de las denominaciones asignadas a los clústeres fueran las siguientes (el orden no implica jerarquía alguna):

Clúster 1 - Competencias profesionales

Clúster 2 - Competencias intra-personales

Clúster 3 - Competencias inter-personales

Clúster 4 - Responsabilidad Social y sentido transcendente
Clúster 1 - Competencias profesionales: 24 ideas con una valoración promedio de $3,83 / 5$

\begin{tabular}{|c|l|c|}
\hline & Formación completa de la persona en aspecto técnico, & \\
\hline 5 & Competencial y personal & 4,55 \\
\hline 15 & Profundidad & 4,27 \\
\hline 55 & Capaces de tomar de decisiones en horizontes inciertos & 4,18 \\
\hline 96 & Capacidad de discernimiento & 4,18 \\
\hline 35 & Capaces de seguir aprendiendo & 4,18 \\
\hline 51 & Buen profesional & 4,09 \\
\hline 12 & Gente con sentido crítico & 4,09 \\
\hline 38 & Pensamiento profundo & 4,00 \\
\hline 84 & Visión estratégica & 4,00 \\
\hline 14 & Agentes de cambio & 4,00 \\
\hline 53 & Visión de futuro & 3,91 \\
\hline 60 & Saber convivir con los problemas & 3,91 \\
\hline 94 & Bien armado intelectualmente & 3,91 \\
\hline 86 & Saber atender a distintos frentes & 3,91 \\
\hline 52 & Emprendedor & 3,82 \\
\hline 80 & Adaptabilidad & 3,73 \\
\hline 42 & Conocedor de idiomas & 3,73 \\
\hline 82 & Imaginación & 3,55 \\
\hline 13 & Capaces de hacer avanzar algo en la vida & 3,55 \\
\hline 56 & Sin miedo al riesgo & 3,36 \\
\hline 61 & No precipitarse en la resolución de problemas & 3,36 \\
\hline 67 & Capacidad de tomar distancia & 3,27 \\
\hline 83 & Disponibilidad para trabajar en el extranjero & 3,27 \\
\hline & & 3,18 \\
\hline
\end{tabular}

Clúster 2 - Competencias intra-personales: 35 ideas y valoración promedio de $3,89 / 5$

\begin{tabular}{|r|l|l|}
\hline 57 & Liderazgo & 4,36 \\
\hline 20 & Responsable de sus actos profesionales & 4,27 \\
\hline 68 & Lealtad & 4,27 \\
\hline 77 & Actitud de "Magis" & 4,27 \\
\hline 50 & Compasivo & 4,18 \\
\hline 45 & Capacidad de interiorización & 4,09 \\
\hline 65 & Equilibrio interior & 4,09 \\
\hline 40 & Equilibrio psicoafectivo & 4,00 \\
\hline 59 & Sentido común & 4,00 \\
\hline 69 & Saber reconocer los propios errores & 4,00 \\
\hline 76 & Aspiración a la excelencia & 4,00 \\
\hline 63 & Saber asumir críticas & 3,91 \\
\hline 21 & Iniciativa & 3,82 \\
\hline 22 & Creativos & 3,82 \\
\hline 75 & No pactar con la mediocridad & 3,82 \\
\hline 19 & Autoconocimiento & 3,73 \\
\hline 58 & Sacrificio & 3,73 \\
\hline 92 & Consciente de sus debilidades & 3,73 \\
\hline 93 & Capaz de superar los baches & 3,73 \\
\hline 29 & Capaces de aprender del frascaso & 3,64 \\
\hline 47 & Capacidad de resistencia & 3,64 \\
\hline 2 & Proactivos & 3,55 \\
\hline 18 & Exigentes consigo mismo & 3,55 \\
\hline 34 & Colocan el esfuerzo como valor positivo & 3,55 \\
\hline 73 & Generoso con la institución (para la que trabaja) & 3,55 \\
\hline 49 & Optimista & 3,45 \\
\hline 88 & Capacidad de contemplación & 3,45 \\
\hline 95 & Disciplinado & 3,45 \\
\hline 26 & Flexibles & 3,27 \\
\hline 70 & Saber celebrar los éxitos de los demás & 3,27 \\
\hline 74 & No vengativo & 3,18 \\
\hline 28 & Capaz de relativizar & 3,09 \\
\hline 33 & No dejarse llevar por las modas & 3,00 \\
\hline 48 & Paciencia & \\
\hline 27 & Con sentido del humor & \\
\hline & & \\
\hline
\end{tabular}


Clúster 3 - Competencias inter-personales: 18 ideas y valoración promedio de $3,74 / 5$

\begin{tabular}{|c|l|l|}
\hline 81 & Capacidad de diálogo & 4,27 \\
\hline 17 & Sabe trabajar en equipo & 4,18 \\
\hline 44 & Capaz de trabajar con personas distintas a él & 4,18 \\
\hline 87 & Capacidad de escucha & 4,18 \\
\hline 3 & Capacidad de comprender al otro & 4,00 \\
\hline 78 & Utilizar la autoridad con mesura & 4,00 \\
\hline 23 & Capacidades de relacionarse con otros & 3,91 \\
\hline 37 & Ponerse en lugar del otro & 3,82 \\
\hline 90 & Capacidad de conocimiento de las personas & 3,82 \\
\hline 64 & Capacidad de negociación & 3,73 \\
\hline 66 & Capacidad de ver las valias distintas de las personas & 3,73 \\
\hline 98 & Capaz de tratar con todo tipo de personas & 3,73 \\
\hline 32 & Capaces de integrar y rehacios a excluir & 3,55 \\
\hline 71 & Equilibrio en el trato & 3,45 \\
\hline 97 & Sencillez en el trato & 3,45 \\
\hline 43 & Aprecio por las otras culturas & 3,36 \\
\hline 54 & Capacidad de trabajar con personal técnico & 3,09 \\
\hline 72 & Entrar con la del otro para salir con la tuya & 2,82 \\
\hline
\end{tabular}

Clúster 4 - Responsabilidad Social y sentido transcendente: 26 ideas y valoración promedio de 3,95/5

\begin{tabular}{|r|l|l|}
\hline 62 & Respeto profundo por las personas & 4,73 \\
\hline 6 & Honestidad & 4,64 \\
\hline & Toma de decisiones pensando en las implicaciones para & \\
\hline 36 & los "últimos" & 4,45 \\
\hline 39 & Liderazgo de servicio & 4,45 \\
\hline 79 & Capacidad de servicio & 4,45 \\
\hline 85 & Actitud de contribuir al bien de la sociedad & 4,45 \\
\hline 4 & Comprometidos con el entorno y su profesión & 4,36 \\
\hline 8 & Con una gran sensibilidad por la justicia & 4,27 \\
\hline 100 & Preocupado por los pobres & 4,27 \\
\hline 102 & Abiertos a la transcendencia & 4,27 \\
\hline 16 & No todo vale para el éxito & 4,18 \\
\hline 99 & Limpio de corazón & 4,18 \\
\hline 103 & Sensibles al humanismo cristiano & 4,09 \\
\hline 101 & Solidarios & 4,00 \\
\hline 10 & Visión por el bien común & 3,91 \\
\hline & Con una cosmovisión cristiana del mundo de la persona & \\
\hline 41 & y de la historia & 3,91 \\
\hline 30 & Que miren más que su propio ombligo & 3,73 \\
\hline 89 & No buscar su propio amor, querer e interés & 3,73 \\
\hline 91 & Altruista & 3,73 \\
\hline 7 & Preocupados por todo lo que no sea sí mismo & 3,55 \\
\hline 24 & Sensibilidad ecológica en lato sensu & 3,45 \\
\hline 31 & Conocimiento pensamiento social cristiano & 3,45 \\
\hline 9 & Respeto hacia lo diverso & 3,36 \\
\hline 11 & Humanistas & 3,36 \\
\hline \multirow{2}{*}{46} & Sentimiento de pertenencia (a la institución donde se & \\
\hline & ha estudiado) & 3,18 \\
\hline & & \\
\hline
\end{tabular}

\section{Fase 6: Utilización de los mapas.}

El mapa resultante con los nombres de cada grupo de rasgos podía ser considerado como la representación gráfica de un modelo sobre de perfil de los egresados de las escuelas de negocios jesuitas.
De los cuatro grupos obtenidos, el primer clúster hace referencia a las competencias profesionales, apareciendo ideas o rasgos como: formación completa de la persona en los aspectos técnico, competencial y personal; visión global, mundial, compleja y abierta; capaces de tomar decisiones en horizontes inciertos; capaces de seguir aprendiendo; buen profesional; sentido crítico; pensamiento profundo; visión estratégica; agentes de cambio; bien armado intelectualmente; emprendedor; etc. Todos ellos conforman una serie de ítems que combinan dimensiones de capacidad intelectual, competencias cognitivas y una actitud proactiva. Dicho de otro modo, rasgos de personas con conocimiento y capacidad de decidir y actuar, básicos para ser buenos profesionales. La ponderación media del grupo ha sido de 3,83 sobre 5 , siendo la idea más valorada del clúster "la formación completa de la persona en los aspectos técnico, competencial y personal" (4.55/5), la tercera mejor valorada de las 103, y la menos valorada "la disponibilidad para trabajar en el extranjero" $(3,18 / 5)$.

El segundo clúster es el más numeroso (35 ideas) y contiene las ideas vinculadas a las competencias intrapersonales, siendo algunos de los rasgos más destacados: liderazgo; responsable de sus actos profesionales; lealtad; actitud de magis; capacidad de interiorización; equilibrio interior; saber reconocer los propios errores; aspiración a la excelencia; iniciativa; autoconocimiento; etc. El conjunto de ítems de este grupo configura un cuadro de características vinculadas al conocimiento de sí mismo y la maduración personal, a la inteligencia emocional en clave intrapersonal, a la gestión de uno mismo y a una actitud de superación y mejora. En otras palabras, personas equilibradas, con valores y que aspiran a dar lo mejor de sí mismas. La ponderación media del grupo ha sido de 3,69 sobre 5. La idea más valorada $(4,36 / 5)$ es "liderazgo"; la menos valorada es "con sentido del humor” $(2,82 / 5)$.

Las competencias inter-personales es el nombre del tercer clúster, el menos numeroso (18 rasgos) pero el que presenta una mayor coherencia interna, y hace referencia a ideas como: capacidad de diálogo; saber trabajar en equipo; capaz de trabajar con personas distintas; capacidad de escucha; capacidad de comprender al otro, utilizar la autoridad con mesura; capacidad de relacionarse con otros; ponerse en el lugar del otro; capacidad de conocimiento de las personas; capacidad de negociación, etc. El conjunto de rasgos de este grupo están claramente vinculados a la descripción de personas con capacidad relacional, sentido de equipo y dialogantes. El clúster está valorado con una puntuación de 3,74/5. La idea más valorada es la "capacidad de diálogo" $(4,27 / 5)$ mientras que la menos valorada es la de "entrar con la del otro para salir con la suya" (2.82/5), la tercera peor valorada del total de 103 de la lista.

Responsabilidad social y sentido transcendente aparece como nombre del cuarto clúster con una puntuación de 3,95/5. Este clúster es el más valorado en promedio y sus cinco primeros rasgos figuran entre los seis más valorados del conjunto de 103 de la lista. Las ideas o rasgos más destacados son: el respeto profundo por las personas; honestidad; toma de decisiones pensando en las implicaciones para los "últimos"; liderazgo de servicio; capacidad de servicio; actitud de contribuir al bien de la sociedad; comprometidos con el entorno y 
la profesión; con una gran sensibilidad por la justicia; preocupado por los pobres; abiertos a la trascendencia; sensibles al humanismo cristiano; etc. Este clúster es claramente de una naturaleza distinta a los anteriores, agrupando los rasgos que hacen referencia a personas preocupadas por los demás y por la sociedad, solidarias, con vocación de servicio e inspiración cristiana, de alguna manera, personas comprometidas, socialmente responsables y abiertas a la trascendencia. La idea más valorada del grupo y también de la lista de 103 rasgos es la de "respeto profundo por las personas" (4.73/5) mientras que la idea menos valorada, tanto en el clúster como en el conjunto de toda la lista, es la de "mala conciencia" (2,55/5).

Habiendo finalizado la presentación de de los resultados obtenidos siguiendo las seis etapas que establece Trochim (1989), pasamos ya a la parte final de conclusiones.

\section{Conclusiones}

Las conclusiones que pueden extraerse tras la investigación realizada están relacionadas con los resultados obtenidos meduiante la aplicación del concept mapping, una metodología que nos ha revelado su alto potencial para conocer cómo conceptualizan una temática concreta un grupo de expertos o focus group, ofreciendo resultados en forma de mapas conceptuales que posibilitan un análisis clúster y una interpretación que resultan muy útiles y valiosos para conocer y sistematizar el conocimiento experto. En este sentido, haber utilizado esta metodología en este estudio representa una novedad en el sector de la educación universitaria y de las escuelas de negocios, pudiendo considerar sus resultados como una aportación original y novedosa al presentar y ordenar el pensamiento y la valoración de un aspecto esencial para las escuelas de negocios jesuitas según lo expresan sus autoridades institucionales.

Los resultados del CM muestran, en primer lugar, una amplia lista de rasgos (103) que han de caracterizar el perfil de los egresados de las escuelas de negocios jesuitas de UNIJES, según el criterio de sus autoridades (directores y rectores). La amplitud de la lista acordada por el focus group revela una visión integral y holística de la formación que se persigue, atendiendo a múltiples dimensiones de la educación y del desarrollo de las personas: intelectual, profesional, psicológica, ética, social, espiritual, etc. Así mismo, los resultados del CM muestran una agrupación coherente de los 103 rasgos obtenidos del focus group de expertos, obteniéndose 4 clústeres con sentido propio y que reflejan los cuatros ámbitos principales a tener en cuenta en la formación de las escuelas de negocios jesuitas al juicio de sus máximos responsables: las competencias para ser un buen profesional (C1), las competencias personales, tanto intra-personales (C2) como inter-personales (C3) $\mathrm{y}$, finalmente, el conjuntos de rasgos que configuran una persona con sentido de su responsabilidad social y abierta a la trascendencia (C4).

Visto en conjunto, los tres primeros clústeres cubren un marco amplio de rasgos o competencias genéricas y específicas que podrían ser, en su mayoría, comunes a la formación universitaria en cualquier escuela de negocios que tuviera una visión amplia de su quehacer educativo y un buen nivel de desempeño. La existencia de un cuarto clúster (Responsabilidad social y sentido transcendente) permite sobrepasar el nivel anterior al presentar un conjunto de características o rasgos que son propios o específicos de una institución universitaria de inspiración cristiana y, más si cabe, con algunos rasgos genuinamente "jesuíticos", ya sea por utilizar significantes propios de la SJ o por el uso de expresiones "apropiadas" en su acervo cultural, tales como: actitud de magis; liderazgo de servicio; toma de decisiones pensando en las implicaciones para "los últimos"; capacidad de discernimiento; agentes de cambio; no buscar su propio amor, querer e interés; entrar con la del otro para salir con la suya; etc.

Los resultados del CM que revelan los cuatro clústeres obtenidos ponen de manifiesto que para los directivos de escuelas de negocios jesuitas de UNIJES, las personas que egresen de sus centros han de caracterizarse --según el orden de la valoración promedio de los clústeres-- por ser personas comprometidas, socialmente responsables y abiertas a la trascendencia; buenos profesionales, personas con conocimiento y capacidad de decidir y actuar; personas dialogantes, con capacidad relacional y sentido de equipo; $y$, finalmente, personas equilibradas, con valores y que aspiren a dar lo mejor de sí mismas.

Los resultados del CM, en su conjunto, concuerdan claramente con el modelo Ledesma-Kolvenbach, pues reflejan su sentido holístico, integral y de formación de la persona completa, así como sus cuatro dimensiones (utilitas, humanitas, istitia y fides) que puede apreciarse cómo se concretan en una gran mayoría de los rasgos de la lista de ítems fruto del brainstorming. Dicho esto, es reseñable que la cuatridimensionalidad del modelo Ledesma-Kolvenbach no se ajusta a los cuatro clústeres obtenidos en el CM. Si "aterrizamos" con dicho modelo sobre los resultados del CM, es observable la coincidencia de la utilitas con el clúster 1 (competencias profesionales), pero sucede que cuando se busca la relación de la humanitas nos encontramos que se corresponde con los clústeres 2 y 3 (competencias intra-pesonales e inter-personales), quedando el clúster 4 como nítido destinatario, por los rasgos que contiene, de dos de las dimensiones, la iustitia y la fides, siendo este clúster el que tiene la valoración promedio más alta y cuyos cinco primeros rasgos figuran entre los seis más valorados del conjunto de 103 de la lista completa.

Estas conclusiones nos llevan a pensar en la importancia de analizar en qué medida se mueven o no en el mismo plano los textos y modelos institucionales sobre la misión y los objetivos de la formación (perfil del egresado), y la conceptualización más concreta de los mismos por parte de las personas con responsabilidades de dirección (rectores y directores) en las escuelas de negocios jesuitas. La profundización en este análisis abre campo a futuras líneas de investigación, tales como:

1. Analizar comparativamente, utilizando la metodología de encuesta, cuál es la valoración y aplicación práctica de los resultados obtenidos en este estudio en otros niveles de las instituciones analizadas: decanos, directores académicos o de programas, profesorado, staff, alumnos y participantes. 
2. Analizar, utilizando el concept mapping y/o la encuesta, cómo conceptualizan el perfil que ha de caracterizar al egresado de las escuelas de negocios sus principales stakeholders: antiguos alumnos, mundo profesional, empresas y reclutadores, instituciones públicas, partners académicos, etc.

3. Analizar cuál es la política institucional y los procedimientos de declaración, aseguramiento y evaluación de la misión que siguen las escuelas de negocios y, más concretamente, aquéllas que se presentan como instituciones mission-driven.

\section{Referencias}

Abell, D.F. (1980). Defining the Business: The starting point of strategic-planning. Prentice Hall, Englewoods Cliffs.

Agúndez, M. (2008a). Discursos Universitarios. P. Peter - Hans Kolvenbach. Edita UNIJES-Provincia de España de la Compañía de Jesús.

Agúndez, M. (2008b). El paradigma universitario Ledesma-Kolvenchbah. Revista Fomento Social 63, 603 - 631. Córdoba.

Arrupe P. (1981). La identidad del Jesuita en nuestros tiempos. Sal Terrae. Santander.

Arrupe, P. (1983). Hombres para los demás. Diafora. Barcelona.

AACSB International. 2015. Eligibility Procedures and Accreditation Standards for Business Accreditation, last retrieved: April 9, 2015, from: www.aacsb.edu/accreditation

Berleur, J., Harvanek, R.F. y Corliss, J.W. (1997). Analysis of Mission Statements or similar Documents of Jesuit Universities and Higher Education Institution. http://www.info.fundp.ac.be/jbl/nis-stat/index.htm

Bigné, J.E., Aldás-Manzano, J., Küster, I. \& Vila, N. (2002). The concept mapping approach in marketing: an application in the travel agencies sector. Qualitative Market Research: An International Journal, 5(2), 87-95.

Campbell, A. y Yeung, S. (1991). Creating a sense of mission. Long Range Planning 24(4), 10-20.

Compañía de Jesús. (1975). Congregación General XXXII. Razón y Fe. Madrid.

Compañía de Jesús. (1995). Congregación General 34. Mensajero. Bilbao.

Collins, J.C. y Porras, J.I. (1996, September-October). Building your company's vision. Harvard Business Review.

David, F.R. (1989). How companies define their mission. Long Range Planning, 22(1), 90-97.

Davies, S.W. and Glaister, K.W. (1997). Business school mission statements - the bland leading the bland? Long Range Planning 30, 594604.
Delbecq, A. (1983). En colaboración con los decanos de la Jesuit Association of Collegiate Schools and Programmes in Business Administration. Jesuit Business Schools: four reflections. Universidad de Santa Clara (EUA).

Drucker, P.F. (1973). Management: Tasks, Responsibilities, Practices. Harper and Row, New York.

Dunn, W.N. (1982). Reforms as argument. Knowledge: Creation, Diffusion, Utilizations, 3, 293-326.

Edem, A. Spencher, P. \& Eyfield, B. (2003). Provides missions and their development. Learning and skills Development Agency. London.

Gil Coria, E. (1999). La pedagogía de los jesuitas, ayer y hoy. Publicaciones de la Universidad Pontificia de Comillas. Madrid.

Guerras L.A. and Navas J.E. (2007). La Dirección Estratégica de la empresa. Teoría y aplicaciones. Thomson.

Guichard, A. (1974). Los Jesuitas. Dopesa Barcelona.

Globally Responsible Leadership Initiative (GLRI) (2008). An initiative by the European Foundation of Management Development (EFMD) with the support of the United Nations Global Compact (UNGC), last retrieved: January 7, 2015, from: https://www.unglobalcompact.org/ docs/news_events/9.1_news_archives/2005_10_18/efmd_rep_grli.pdf

Hair, J.F, Black, B., Babin, B., Anderson, R.E., \& Tatham, R.L. (2006). Multivariate data analysis (6th Edition). New Jersey: Prentice Hall.

Johnson, G. and Scholes, K. (1997). Dirección Estratégica. Análisis de la estrategia de las organizaciones. Prentice Hall.

Khurana, R. (2007). From Higher Aims to Hired Hands: The Social Transformation of American Business Schools and the Unfulfilled Promise of Management as a Profession, Princeton University Press.

Lacouture, J. (1994). Jesuitas. Ediciones Paidós. Barcelona. Vol. 1 y 2.

Lowney, C. (2005). El liderazgo al estilo de los jesuitas. Las mejores prácticas de una compañía de 450 años que cambió el mundo. Ediciones Granica. Barcelona.

Losada, C., Martell, J., \& Lozano, J. M. (2011). Responsible business education: Not a question of curriculum but a raison dêtre for business schools, in Morsing and Sauquet (Eds.), Business Schools and their Contribution to Society, Sage Publications, pp. 163-174.

Margenat, J.M. (2010), Competentes, conscientes, compasivos y comprometidos. La educación de los jesuitas. Editorial PPC. Madrid.

Martin, J. (2011). Más en las obras que en las palabras. Una guía (ignaciana) para casi todo. Mensajero- Sal Terrae. Bilbao-Santander.

Mussi, J. M. (2008). Does Mission Matter? Exploring the relationship between the Mission of Jesuit Higher Education and new student affairs professionals. Dissertation. 
Nabitz, U., Severens, P., Brink, W. \& Jansen, P. (2007). Improving the EFQM model: an empirical study on model development and theory building using concept mapping. Total quality management, 12(1), 69-81.

Nicolás, A. (2008). Misión y Universidad. ¿Qué futuro queremos? Conferencia publicada en ESADE, 50 años inspirando futuros, edición de ESADE-URL (2010).

Nicolás, A. (2010). Profundidad, universalidad y ministerio académico. Desafíos a la educación superior jesuita de hoy. Encuentro Mundial de Rectores de Universidades Jesuitas. Universidad Iberoamericana de la Ciudad de México.

O’Malley, J.W. (1995). Los primeros jesuitas. Mensajero- Sal Terrae. Bilbao-Santander.

Osborn, A.F. (1948). Your creative power. New York. Charles Scribner.

Pearce. J.A. (1982). The company mission as a strategic tool. Sloan Management Review. 24, 15-24.

PRME. 2008. A Global Initiative - A Global Agenda. United Nations Global Compact. May 10, 2008, last retrieved: January 7, 2015, from: http://www.unprme.org/resources-and-reporting/resource-docs/ PRMEBrochureFINALlowres.pdf.
Rosas, S.R. \& Camphausen, L.C. (2007). The use of concept mapping for scale development and validation in evaluation. Evaluation and program planning, 30, 125-135.

Salvat, I. (2001). Servir en Misión Universal. Mensajero-Sal Terrae. Bilbao y Santander.

Santomà, R. (2008). Aspectos de gestión en la calidad de servicio. Una aplicación del concept mapping al caso de las cadenas hoteleras en España. Tesis Doctoral.

Starkey, K. and Tempest, S. (2008). A Clear Sense of Purpose? The Evolving Role of the Business School, Journal of Management Development, 27(4), 379-390.

Taylor, S.J. y Bodgan, R. (1987). Introducción a los métodos cualitativos de investigación: la búsqueda de significados. Editorial Paidós Básica. Barcelona.

Trochim, W.M.K (1989). An introduction to concept mapping for planning and evaluation. Evaluation and Program Planning, 12, 1-16.

Woodrow, A. (1985). Los Jesuitas: Historia de un dramático conflicto. Planeta. Barcelona. 